\title{
Discrepancies between Canadian cancer research funding and site-specific cancer burden: a spotlight on ten disease sites
}

\author{
A.C. Coronado MSc, ${ }^{*}$ C. Finley MD, ${ }^{*}$ K. Badovinac MA MBA, ${ }^{*}$ J. Han MPH, ${ }^{*}$ J. Niu MSc, ${ }^{*}$ and R. Rahal $\mathrm{MBA}^{*}$
}

\begin{abstract}
Background Cancer research is essential in evaluating the safety and effectiveness of emerging cancer treatments, which in turn can lead to ground-breaking advancements in cancer care. Given limited research funding, allocating resources in alignment with societal burden is essential. However, evidence shows that such alignment does not typically occur. The objective of the present study was to provide an updated overview of site-specific cancer research investment in Canada and to explore potential discrepancies between the site-specific burden and the level of research investment.

Methods The 10 cancer sites with the highest mortality in 2015-which included brain, female breast, colorectal, leukemia, lung, non-Hodgkin lymphoma, ovary, pancreas, prostate, and uterus-were selected for the analysis. Information about site-specific research investment and cancer burden (raw incidence and mortality) was obtained from the Canadian Cancer Research Survey and Statistics Canada's CANsim (the Canadian Socio-Economic Information Management System) respectively. The ratio of site-specific research investment to site-specific burden was used as an indicator of overfunding (ratio $>1$ ) or underfunding (ratio $<1$ ).
\end{abstract}

Results The 3 cancer sites with the highest research investments were leukemia, prostate, and breast, which together represented $51.3 \%$ of 2015 cancer research funding. Conversely, the 3 cancer sites with the lowest investments were uterus, pancreas, and ovary, which together represented $7.8 \%$ of 2015 research funding. Relative to site-specific cancer burden, the lung, uterus, and colorectal sites were consistently the most underfunded.

Conclusions Observed discrepancies between cancer burden and research investment indicate that some cancer sites (such as lung, colorectal, and uterus) seem to be underfunded when site-specific incidence and mortality are taken into consideration.

Key Words Research investment, incidence, mortality

Curr Oncol. 2018 Oct;25(5):338-341

www.current-oncology.com

\section{INTRODUCTION}

Cancer research is essential in evaluating the safety and effectiveness of emerging cancer treatments. For example, clinical trials lead to the development of new and moreeffective treatments for specific types of cancer, less-toxic anticancer drugs, and less-invasive methods of surgery and tumour resection ${ }^{1}$. Research investment for specific disease sites has resulted in advances in anticancer treatments and early detection methods, improving patient survival and lessening the overall burden of specific cancers.
Ideally, research funding targeting specific cancer sites should be distributed in alignment with the associated societal burden (for example, site-specific mortality, lifeyears lost). However, evidence shows that such alignment is not the case in developed countries. A study of research funding in the United States showed that some cancers (leukemia, and breast and prostate cancer) are funded at levels higher than their relative burden and that others were considerably underfunded, including lung, bladder, and kidney cancer ${ }^{2}$. Similar findings were reported in a recent study from the United Kingdom, which examined research 
funding for 26 cancer sites in relation to site-specific burden. The authors highlighted the critical situation of lung cancer in terms of funding: despite being responsible for $26 \%$ of cancer deaths, lung cancer received $6 \%$ of total site-specific research funding in $2010^{3}$.

The Canadian cancer research funding landscape might be similar. In 2005, research investments in prostate, colorectal, and lung cancer were significantly lower than those in breast cancer, even though prevalence was evenly distributed ${ }^{4}$. Also, the numbers of research and clinical trial publications are lower for lung, colorectal, and pancreatic cancers than for other disease sites, despite the fact that the former three sites have the highest mortality rates ${ }^{5}$. Such disparities highlight the need for an exploration of gaps in research investment and for the formulation of evidence-based strategies to maximize the impact of research dollars on cancer control.

The present study provides an updated overview of the distribution of Canadian cancer research funding relative to the cancer burden for the 10 disease sites with the highest cancer mortality and highlights potential discrepancies between site-specific burden and level of research investment. It is fundamental to clarify that the objective of the study is not to discourage funding of specific cancer sites in an era in which annual cancer research funding is declining. Rather, the intention is to shed light on disease sites that have remained in the shadows in terms of research investments despite imposing the highest cancer burden on Canadians.

\section{METHODS}

Site-specific information was obtained for the 10 cancer disease sites with the highest number of cancer deaths in 2015 (the latest year for which mortality and research investment data were available). The sites included were brain, female breast, colorectal, leukemia, lung, nonHodgkinlymphoma, ovary, pancreas, prostate, anduterus.All other disease sites were categorized as "other." Information about site-specific cancer research investments was retrieved from the Canadian Cancer Research Survey, which is an annual survey developed by the Canadian Cancer ResearchAlliance thatsystematicallycollectscancerfunding information from 42 organizations and programs across the country (https://www.ccra-acrc.ca/index.php/initiatives /canadian-cancer-research-survey). The information collected was harmonized, de-duplicated, and coded to disease site and area of science (International Cancer Research Partnership's Common Scientific Outline).

To indicate the burden of cancer, raw cancer incidences (number of new cases) and mortality values (number of cancer deaths) were obtained from Statistics Canada's CANSIM (Canadian Socio-Economic Information Management System) tables. Cancer-site definitions for retrieving incidence and mortality were aligned with Canadian Cancer Society's descriptions.

To estimate the extent of underfunding or overfunding relative to the site-specific cancer burden (incidence, mortality cases), we calculated the ratio of site-specific research investment to site-specific burden as described by Carter and Nguyen ${ }^{2}$. That ratio is calculated as the amount of site-specific research funding expressed as the percentage of overall funding divided by the site-specific cancer burden (expressed as the percentage of raw incidence or mortality). A ratio greater than 1 is interpreted as a general indication of overfunding, and a ratio less than 1 is indicative of underfunding.

\section{RESULTS}

In 2015, the total annual cancer research investment was $\$ 480,174,971$ - the lowest in the preceding 5 years (data not shown). Of that total, $\$ 284,046,935$ (59.2\%) was allocated to specific cancer sites. The remaining amount was invested in research relevant to all cancers. Figure 1 presents an overview of the site-specific disease burden (percentage of incident cases and mortality) and the distribution of research funds by cancer site. In terms of cancer burden, the highest proportions of new cancer cases and deaths occurred for the lung, breast, and colorectal sites. The percentage of research investment varied substantially by cancer site, ranging from $0.6 \%$ (uterus) to $22.5 \%$ (breast).

The 3 cancer sites with the highest research investments were leukemia, prostate, and breast, which together represented $51.3 \%$ of 2015 cancer research funding, $26.8 \%$ of new cancer cases, and $15.4 \%$ of cancer-related deaths (Figure 1). Conversely, the 3 cancer sites with the lowest investments were uterus, pancreas, and ovary, which together represented $7.8 \%$ of 2015 research funding, $7.0 \%$ of new cancer cases, and $10.3 \%$ of cancer deaths. It is worth noting that lung cancer had the highest proportion of new cancer cases (13.3\%) and deaths $(26.1 \%)$, but received only $5.0 \%$ of 2015 research funding.

Discrepancies between funding level and site-specific cancer burden are reflected in Figure 2, which presents the ratio of the site-specific research investment (percentage relative to total 2015 funding) to site-specific incidence [percentage relative to overall cancer incidence; Figure 2(A)] and mortality [percentage relative to overall cancer mortality; Figure 2(B)]. A ratio less than 1 is a general indication of underfunding, and a ratio greater than 1 can be interpreted as overfunding. When site-specific incidence was considered, the top 3 overfunded cancer sites were ovary, leukemia, and brain. Considering cancer deaths, the top 3 overfunded sites were prostate, breast, and leukemia. Although different results are commonly expected depending on the selected denominator, the lung, uterus, and colorectal sites were consistently the most underfunded.

\section{DISCUSSION}

Sustained investments are critical to expand research capacity for specific cancers, to achieve advancements in patient care, and to refine technologies that can be implemented in the broader health care system. Over time, new approaches to cancer care can result in improved outcomes. For instance, the decline in breast cancer mortality observed since the early 1990s in developed countries has been attributed to substantial improvements in systemic therapies and advancements in early cancer detection, catalyzed by investments in breast cancer research ${ }^{6,7}$. 


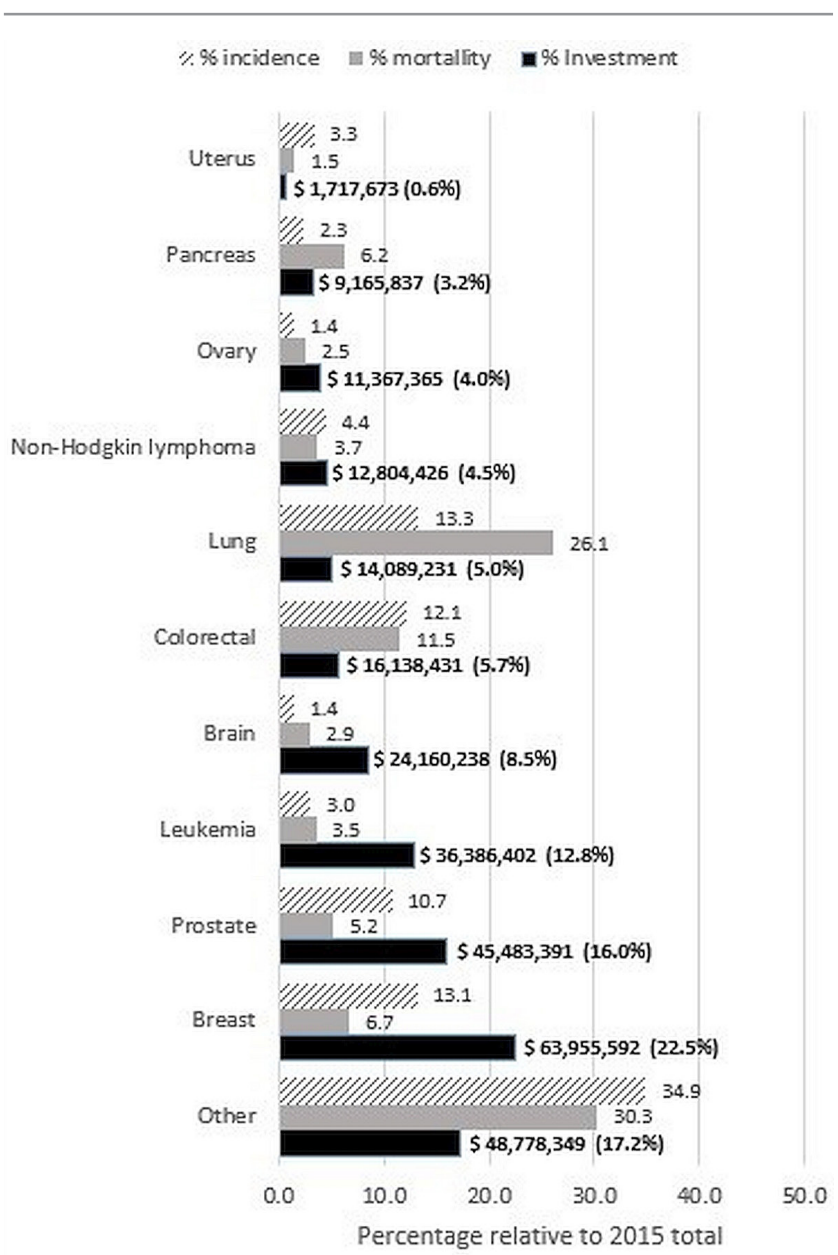

FIGURE 1 Site-specific cancer incidence, mortality, and research investment, 2015.

Although research efforts should ideally be aligned with disease burden, results indicate that such alignment might not be the case for some cancers in Canada. In 2015, lung, colorectal, and breast cancer imposed the highest burden on Canadians in terms of cancer incidence and mortality. With the exception of breast cancer, those disease sites were not highly funded. Colorectal cancer and lung cancer received, respectively, $5.7 \%$ and $5.0 \%$ of 2015 research funding even though, together, they were responsible for more than one third of cancer-related deaths. That mortality exceeded breast cancer mortality by a factor of 6 , and yet comparatively, the proportion of research investment that they received was less than half the research funding allocated to breast cancer. The ratios shown in Figure 2 reflect those gaps and also reveal research funding discrepancies in less-common cancer sites, including uterus and pancreas.

Studies from other countries show similar research funding patterns. A U.K. analysis identified the leukemia, ovary, and cervix sites as overfunded, and the lung, pancreas, esophagus, and bladder sites as underfunded ${ }^{8}$. A U.S. study analyzed research funding allocation from the U.S. National Cancer Institute and found that the breast, prostate, and leukemia sites were overfunded relative to the
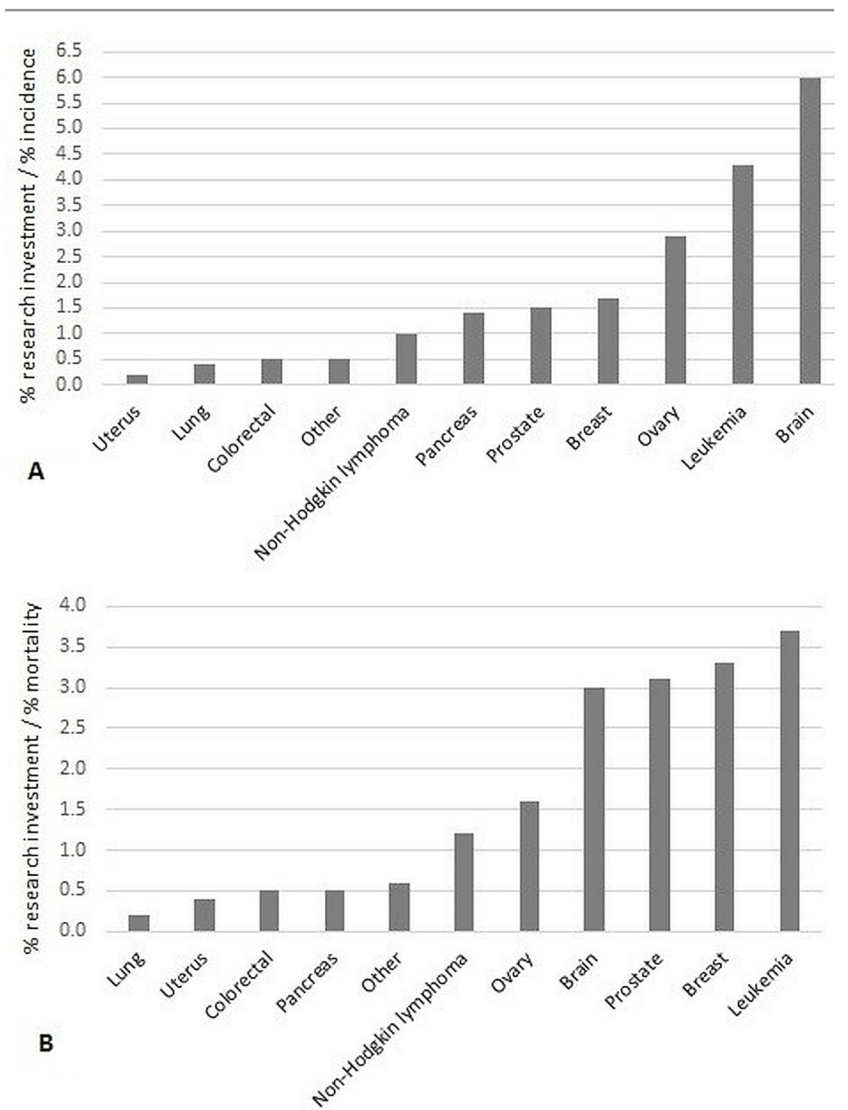

FIGURE 2 Cancer research funding relative to site-specific burden. The numerator is the site-specific research funding expressed as a percentage of total 2015 funding, divided by (A) the site-specific incidence (percentage relative to overall cancer incidence) and (B) the site-specific mortality (percentage relative to overall cancer mortality). A ratio less than 1 is indicative of underfunding.

societal burden of other cancer sites. Conversely, the liver, bladder, esophagus, oral, pancreas, stomach, and uterus sites were relatively underfunded. In that analysis, lung cancer was considered an outlier because of the extreme gap between its health burden and level of research investment ${ }^{2}$. Interestingly, colorectal cancer was not identified as underfunded in any of the foregoing studies.

Variables other than disease burden also influence funding decisions. Research feasibility, technology costs, infrastructure and skill requirements, and political and social considerations also play a role in the funding allocation process. Those factors could explain the observed discrepancies between the burden of common, high-mortality cancers and the level of research funding received. Advocates of underfunded disease sites facing those challenges could maximize their research funding options if organizations develop a common agenda to target efforts that could secure research investments.

\section{Limitations}

A common limitation of research funding studies is lack of publicly available research investment information, mostly because pharmaceutical company information is confidential, and those entities are not required to disclose research 
expenses. As previously mentioned, it is estimated that the Canadian Cancer Research Survey covers $60 \%-80 \%$ of the overall cancer research funding in Canada. However, it does not include institution-specific funding from hospital foundations, research supported by private foundations, or industry research and development (unless such funding forms a part of project partnerships captured in the survey).

Many metrics are available to estimate the societal or health burden of a disease. Site-specific incidence and mortality metrics have the advantage of being the most feasible first step in an exploration of research funding discrepancies, and they are easy to interpret. It is recommended that future studies use additional measures of health burden, including life-years lost and disabilityadjusted years, among others.

\section{CONCLUSIONS}

With annual cancer research funding in decline, only the objective identification of illnesses that receive nonoptimal investment can allow for adjustments that might minimize the overall health costs of disease in Canada. The observed discrepancies between cancer burden and research investment indicate that some cancer sites (such as lung, colorectal, and uterus) seem to be underfunded when the metrics of their burden (such as incidence and mortality) are taken into consideration. If Canada is committed to making advancements in cancer care for individuals affected by cancer today and to improving cancer control for future generations, a boost in research capacity for lung and colorectal cancers, which are responsible for $25.4 \%$ of new cancer cases and $37.6 \%$ of cancer-related deaths, is overdue.

Surveillance and monitoring of our Canadian cancer system is of vital importance to identify strategies that will reduce the impact of cancer on Canadians. This autumn, the System Performance Initiative at the Canadian
Partnership Against Cancer will be releasing the 2018 Cancer System Performance Report, which includes data about trends in adult cancer clinical trial participation and long-term outcomes. More information about the Partnership's system performance reports can be found at http://systemperformance.ca/.

\section{CONFLICT OF INTEREST DISCLOSURES}

We have read and understood Current Oncology's policy on disclosing conflicts of interest, and we declare that we have none.

\section{AUTHOR AFFILIATIONS}

${ }^{*}$ Canadian Partnership Against Cancer, Toronto, and ${ }^{\dagger}$ Department of Surgery, McMaster University, Hamilton, ON.

\section{REFERENCES}

1. Canadian Partnership Against Cancer (CPAC). The 2016 Cancer System Performance Report. Toronto, ON: CPAC; 2016.

2. Carter AJ, Nguyen CN. A comparison of cancer burden and research spending reveals discrepancies in the distribution of research funding. BMC Public Health 2012;12:526.

3. Carter AJR, Delarosa B, Hur H. An analysis of discrepancies between United Kingdom cancer research funding and societal burden and a comparison to previous and United States values. Heal Res Policy Syst 2015;13:62.

4. Branton PE. Does Canadian research investment relate to cancer burden? Lancet Oncol 2008;9:82-3.

5. Patafio FM, Brooks SC, Wei X, Peng Y, Biagi J, Booth CM. Research output and the public health burden of cancer: is there any relationship? Curr Oncol 2016;23:75-80.

6. Jatoi I, Miller AB. Why is breast-cancer mortality declining? Lancet Oncol 2003;4:251-4.

7. Bosetti C, Bertuccio P, Levi F, Chatenoud L, Negri E, La Vecchia C. The decline in breast cancer mortality in Europe: an update (to 2009). Breast 2012;21:77-82.

8. Maruthappu M, Head MG, Zhou CD, et al. Investments in cancer research awarded to U.K. institutions and the global burden of cancer 2000-2013: a systematic analysis. BMJOpen 2017;7:e13936. 